\title{
Electrophysiology of Memory-Updating Differs with Age
}

\author{
Genevieve Z. Steiner ${ }^{1,2 *}$, Craig J. Gonsalvez ${ }^{3}$, Frances M. De Blasio ${ }^{2}$ and Robert J. Barry ${ }^{2}$ \\ ${ }^{1}$ The National Institute of Complementary Medicine (NICM), Western Sydney University, Penrith, NSW, Australia, ${ }^{2}$ Centre for \\ Psychophysics, Psychophysiology, and Psychopharmacology, Brain \& Behaviour Research Institute, and School of \\ Psychology, University of Wollongong, Wollongong, NSW, Australia, ${ }^{3}$ School of Social Sciences and Psychology, Western \\ Sydney University, Penrith, NSW, Australia
}

OPEN ACCESS

Edited by:

Lutz Jäncke,

University of Zurich, Switzerland

Reviewed by:

Rongqiao He,

Chinese Academy of Sciences, China Juliana Yordanova,

Bulgarian Academy of Sciences,

Bulgaria

*Correspondence:

Genevieve Z. Steiner

G.Steiner@westernsydney.edu.au

Received: 17 March 2016

Accepted: 27 May 2016

Published: 16 June 2016

Citation:

Steiner GZ, Gonsalvez CJ, De Blasio FM and Barry RJ (2016) Electrophysiology of Memory-Updating Differs with Age.

Front. Aging Neurosci. 8:136. doi: 10.3389/fnagi.2016.00136
In oddball tasks, the P3 component of the event-related potential systematically varies with the time between target stimuli-the target-to-target interval (TTI). Longer TTls result in larger P3 amplitudes and shorter latencies, and this pattern of results has been linked with working memory-updating processes. Given that working memory and the P3 have both been shown to diminish with age, the current study aimed to determine whether the linear relationship between $\mathrm{P} 3$ and $\mathrm{TT}$ is compromised in healthy aging by comparing $\mathrm{TT}$ effects on P3 amplitudes and latencies, and reaction time (RT), in young and older adults. Older adults were found to have an overall reduction in P3 amplitudes, longer latencies, an anterior shift in topography, a trend toward slower RTs, and a flatter linear relationship between $\mathrm{P} 3$ and $\mathrm{TI}$ than young adults. Results suggest that the ability to maintain templates in working memory required for stimulus categorization decreases with age, and that as a result, neural compensatory mechanisms are employed.

Keywords: P3(00), aging, working memory, event-related potentials (ERPs), older adults, healthy aging, target-totarget interval (TTI), oddball task

\section{INTRODUCTION}

\section{Electrophysiology of Memory-Updating Differs with Age}

Working memory, the ability to hold and manipulate information in the mind, decreases across the lifespan (Hedden and Gabrieli, 2004), particularly after 70 years of age (Hester et al., 2004). This age-related cognitive decline can result in a loss of confidence, independence, and reduced quality of life. The decline in working memory ability is thought to result from a reduction in regional brain volume and cortical thickness due to a loss of synaptic density, particularly in the prefrontal cortex (Kadota et al., 2001; Pieperhoff et al., 2008; Salat et al., 2009; Bishop et al., 2010). In order to devise strategies to mitigate the pathophysiology of the aging process, an increased understanding of age-related changes in brain function is required.

The P3(00) component of the human event-related potential (ERP), a large positive deflection elicited by salient stimuli, has been described as an electrophysiological index of working memory processes (Squires et al., 1976, 1977; Polich, 1997). Previous work has shown that by extending the time between target stimuli (the target-to-target interval [TTI]), the P3 increases in amplitude and decreases in latency (Gonsalvez et al., 1999, 2007; Gonsalvez and Polich, 2002; Croft et al., 2003; Steiner et al., 2013a,b; Steiner et al., 2014a,b). This "TTI effect" on the P3 has been directly linked

Abbreviations: EEG, Electroencephalogram; EOG, Electrooculogram; ERPs, Event-related potentials; MCI, Mild Cognitive Impairment; RT, Reaction time; TTI, Target-to-target interval. 
with working memory processes (Gonsalvez et al., 2007), where young adults with better working memory ability showed a steeper increase in P3 amplitude with TTI than those with poorer working memory (Steiner et al., 2013a).

The template-update model has been proposed as a framework to account for the TTI effect on the P3 (Gonsalvez et al., 2007). Here, a stimulus activates a profile, or template, of neural activity in working memory that degrades over time. The template will be updated when the stimulus is next presented (longer TTIs = greater update). In the template-update model, a greater increase in P3 amplitude with longer TTIs indicates more effective activation of working memory processes.

To date, the TTI effect has been explored only in young adults. To address this, the present study examined the TTI effect in older adults, and whether the linear relationship between P3 and TTI would vary with age. It was hypothesized that a flatter linear relationship (P3 as a function of TTI) would be observed for older adults, interpreted by the template-update model as indicating less effective electrophysiological memory-updating processes.

\section{METHODS}

\section{Participants}

Participants were 19 students (young adults; mean age $=21.2$, $S D=3.7$, range $=18-35$ years, 14 females, 18 right-handed) from the University of Wollongong and 22 older adults (mean age = $68.1, S D=4.2$, range $=59-74$ years, 16 females, 21 right-handed) from an independent living aged-care facility. Young adults received course credit for participating, and older adults were reimbursed $\$ 40$ (Australian) for their time. Prior to commencing the experiment, participants provided informed consent, and were free to withdraw at any time without penalty. Individuals self-reporting neurological or psychiatric illnesses, and/or use of psychotropic medication, were excluded. It should be noted that although care was taken to exclude participants taking psychoactive medications (e.g., selective serotonin reuptake inhibitors), most older adults took a range of medications for various other health conditions, for example perindopril, celecoxib, rani 2, nonsteroidal anti-inflammatories, lomotil, diabex, esomeprazole, glucosamine etc. Older participants were also screened for cognitive impairment with the Rowland Universal Dementia Assessment Scale (RUDAS; Conforti et al., 2006); all scored $>22$ point cut-off $(M=28.0, S D=1.5)$. Self-reports indicated that participants had refrained from psychoactive substances for at least $12 \mathrm{~h}$ and from tea, coffee, alcohol, and cigarettes for at least $2 \mathrm{~h}$ prior to testing. All participants had normal or corrected-to-normal vision and selfreported normal hearing.

\section{Procedure}

A demographic and screening questionnaire was completed by all participants before they were fitted with electroencephalogram (EEG) recording apparatus. Prior to the experiment, participants completed an electrooculogram (EOG)/EEG calibration task (Croft and Barry, 2000). Participants were seated 600-800 $\mathrm{mm}$ in front of a $48.3 \mathrm{~cm}\left(19^{\prime \prime}\right)$ Dell LCD monitor and instructed to fixate on a $10 \times 10 \mathrm{~mm}$ black cross centered on a gray background.
The experimental task was a visual oddball paradigm (target probability $=30 \%$, nontarget probability $=70 \%$ ) broken into four different blocks (approximately $3 \mathrm{~min}$ each), with short rest intervals between blocks to minimize fatigue. Stimuli consisted of $45 \times 45 \mathrm{~mm}$ black "tick" (target) and "cross" (nontarget) images, each presented for $300 \mathrm{~ms}$ on a gray background with a fixed $1.5 \mathrm{~s}$ ISI. The TTI was manipulated $1.5-12.0 \mathrm{~s}$, with 30 trials presented for each TTI of interest $(1.5,3.0,6.0$, and $9.0 \mathrm{~s})$; a total of 532 stimuli were presented in a randomized sequence. To balance possible speed/accuracy trade-offs, participants were instructed to "respond to target stimuli with a button press, as quickly and as accurately as possible." Participants responded with their dominant hand on a Logitech ${ }^{\circledR}$ Precision game controller. Instruction was given to sit as still as possible, but participants were not directly instructed to refrain from blinking (Verleger, 1991). This procedure was approved by the joint South Eastern Sydney/Illawarra Area Health Service and University of Wollongong Health and Medical Human Research Ethics Committee.

\section{Materials and Apparatus}

EEG data were recorded continuously DC-70 Hz from A2 and 19 scalp sites (Fp1, Fp2, F7, F3, Fz, F4, F8, T3, C3, Cz, C4, T4, T5, P3, Pz, P4, T6, O1, O2) with an electrode cap using tin electrodes, referenced to $\mathrm{A} 1$. The cap was grounded by an electrode located midway between Fp1/Fp2 and Fz. Data were acquired using a Neuroscan Synamps 2 digital signal-processing system and Neuroscan 4.3.1 Acquire software, and the display and stimulus markers were controlled by a linked stimulus computer using Neurobehavioral Systems Inc. Presentation V 13.0 Build 01.23.09 software.

EOG was recorded using tin cup electrodes placed $2 \mathrm{~cm}$ above and below the left eye for vertical movements, and on the outer canthus of each eye for horizontal movements. Impedance was less than $5 \mathrm{k} \Omega$ for cap, EOG, and reference electrodes. Scalp and EOG potentials were amplified with a gain of 500 and digitized at a rate of $1000 \mathrm{~Hz}$.

\section{Data Extraction}

The EEG data were EOG corrected using the RAAA EOG Correction Program (Croft and Barry, 2000). Single trial ERPs were re-referenced to digitally linked ears and extracted offline using the Neuroscan Edit software, low pass filtered (0.1-30 $\mathrm{Hz}$, zero-phase shift, $24 \mathrm{~dB} /$ Octave), epoched -100-900 ms, and baseline corrected using the $100 \mathrm{~ms}$ pre-stimulus period. Trials containing omission (misses) or commission (false alarms) errors, or response times (RTs) longer than $800 \mathrm{~ms}$, were excluded. Data were manually inspected for additional artifacts, and any contaminated trials were rejected, together with errors and RTs $>800 \mathrm{~ms}$. An average of $2.12 \%$ trials $(S D=0.95)$ were excluded for young adults, and $2.02 \%$ trials $(S D=0.95)$ for older adults; these did not differ significantly $t_{(39)}=0.313, p=0.756$. For each subject, averages were computed for each of the four TTIs of interest. P3 peak amplitudes and latencies were obtained from these means for each subject and each of the TTIs, relative to the $100 \mathrm{~ms}$ pre-stimulus baseline; P3 amplitudes and latencies were also computed for nontarget means for each subject. Peak 
data were automatically selected and manually checked using a latency window of 280-420 ms for young adults, and 280-450 ms for older adults. These latency windows were decided based on the grand mean ERPs.

\section{Statistical Analyses}

The design was mixed, with a between-subjects factor of group (young vs. older) and within-subjects factor of TTI (1.5, 3.0, 6.0 , and $9.0 \mathrm{~s}$ ). Separate MANOVAs were carried out on P3 amplitudes and latencies with the above factors, with planned orthogonal contrasts to assess topography across the midline sites ( $\mathrm{Fz}$ vs. $\mathrm{Pz}$, and $\mathrm{Cz}$ vs. mean of $\mathrm{Fz}$ and $\mathrm{Pz}$ ). Topographic distribution of P3 amplitudes and latencies can be examined efficiently by utilizing these orthogonal planned contrasts. Trends over TTI were assessed within subjects, using orthogonal polynomial contrasts with weighted linear and quadratic trends. The effect of stimulus type (target vs. nontarget) was examined by collapsing across TTI and using mixed MANOVAs between groups and across the midline sites separately for P3 amplitudes and latencies. Mixed MANOVA examined the effect of group and interval on RT; again with weighted linear and quadratic trend analyses. No Bonferroni-type $\alpha$ adjustment was required as $a$ priori contrasts were used, and the number of contrasts did not exceed the degrees of freedom for effect (Tabachnick and Fidell, 1989). We also tested for any correlations between our three outcome measures (P3 amplitude and latency, and RT), with each subject and TTI as data points, separately for each group; Fisher $z$ transformations were then calculated to compare groups. All $F$-tests reported have $(1,39)$ degrees of freedom.

It should also be noted that, as this paper details results for a number of dependent measures, the frequency of Type I errors increases. However, this increase in frequency of Type I errors cannot be controlled by adjusting $\alpha$-levels, because the probability of Type I error remains the same (Howell, 1997).

\section{RESULTS}

To aid interpretation of results, trends analyzed across TTIs are denoted as "linear TTI" and "quadratic TTI." The direction of difference between variables is indicated by " $<$ " and " $>$ ", and interactions between effects by " $x$ ". Table 1 shows the relative change (\%) in P3 amplitudes, latencies, and RTs $c f$. the $1.5 \mathrm{~s}$ TTI level, separately for young and older adults.

\section{Grand Means}

Figure 1 illustrates the grand mean ERPs for targets and nontargets from midline sites for young (solid line) and older (dashed line) adults. Grand mean ERPs for each of the four TTIs of major interest from midline sites are displayed in Figure 2 (young adults: left column; older adults: right column). Figure 3 shows grand mean headmaps for young (left) and older (right) adults, separately for targets (top) and nontargets (bottom); mean P3 amplitudes were taken at the midpoint of the latency window for young (350 ms) and older (365 ms) adults.
TABLE 1 | Percentage change in P3 amplitudes $(\mu \mathrm{V})$ at Pz, latencies (ms) at $\mathrm{Cz}$, and RTs (ms) to targets relative to the first TTI (1.5 s), separately for young and older adults.

\begin{tabular}{lcccc}
\hline & \multicolumn{3}{c}{ TTI } \\
\cline { 2 - 5 } & $\mathbf{1 . 5} \mathbf{s}$ & $\mathbf{3 . 0} \mathbf{s}$ & $\mathbf{6 . 0} \mathbf{~ s}$ & $\mathbf{9 . 0 ~ \mathbf { ~ s }}$ \\
\hline YOUNG & & & \\
Amplitude & $100 \%$ & $123 \%$ & $126 \%$ & $131 \%$ \\
Latency & $100 \%$ & $101 \%$ & $99 \%$ & $102 \%$ \\
RT & $100 \%$ & $95 \%$ & $94 \%$ & $95 \%$ \\
OLDER & & & & \\
Amplitude & $100 \%$ & $113 \%$ & $113 \%$ & $113 \%$ \\
Latency & $100 \%$ & $100 \%$ & $97 \%$ & $98 \%$ \\
RT & $100 \%$ & $97 \%$ & $96 \%$ & $94 \%$ \\
\hline
\end{tabular}

\section{P3 Amplitude}

Across group and TTI, P3 was larger at $\mathrm{Pz}$ than $\mathrm{Fz}(\mathrm{Pz}>\mathrm{Fz}: F=$ 230.08, $\left.p<0.001, \eta_{p}^{2}=0.86\right)$, and at $\mathrm{Cz}$ compared the mean of $\mathrm{Fz}$ and $\mathrm{Pz}\left(\mathrm{Cz}>\right.$ mean Fz/Pz: $\left.F=6.23, p=0.017, \eta_{p}^{2}=0.14\right)$. These parietal and central enhancements were greater for targets than nontargets $(\mathrm{Pz}>\mathrm{Fz} \times$ target $>$ nontarget: $F=124.29, p<0.001$, $\eta_{p}^{2}=0.76 ; \mathrm{Cz}>$ mean $\mathrm{Fz} / \mathrm{Pz} \times$ target $>$ nontarget: $F=10.14, p=$ $\left.0.003, \eta_{p}^{2}=0.21\right)$, and this contributed to a main effect of stimulus type (target $>$ nontarget: $F=20.48, p<0.001, \eta_{p}^{2}=0.34$ ). At Pz, P3 was larger for young compared to older adults $(\mathrm{Pz}>\mathrm{Fz} \times$ young $>$ older: $\left.F=31.09, p<0.001, \eta_{p}^{2}=0.44\right)$. Headmaps in Figure 3 also demonstrate that P3 was slightly more frontal for older than young adults, particularly for nontargets.

Figure 4 (upper) illustrates target P3 amplitudes as a function of TTI at Pz, separately for the young and older groups. Across groups and sites, P3 amplitudes increased as TTI increased (linear TTI: $F=17.57, p<0.001, \eta_{p}^{2}=0.31$ ), this plateaued at longer intervals (quadratic TTI: $F=5.44, p=0.025, \eta_{p}^{2}=0.12$ ). The linear increase was most apparent at $\mathrm{Pz}$ (linear TTI $\times \mathrm{Pz}>$ Fz: $\left.F=8.08, p=0.007, \eta_{p}^{2}=0.17\right)$ and $\mathrm{Cz}$ (linear TTI $\times \mathrm{Cz}>$ mean Fz/Pz: $F=21.36, p<0.001, \eta_{p}^{2}=0.35$ ). Figure 4 (upper) shows that the increase in P3 amplitudes with TTI increments was greater for young than older adults (linear TTI $\times$ young $>$ older: $\left.F=13.05, p=0.001, \eta_{p}^{2}=0.25\right)$, this effect was largest at $\mathrm{Pz}$ (linear TTI $\times$ young $>$ older $\times \mathrm{Pz}>$ Fz: $F=4.20, p=0.047$, $\left.\eta_{p}^{2}=0.10\right)$. This can been seen as a relative change in Table 1 , where P3 amplitude is stable for older adults after the second TTI level, but it continues to increase over intervals for young adults.

\section{P3 Latency}

$\mathrm{P} 3$ latencies were longest at $\mathrm{Cz}$, compared to the mean of $\mathrm{Fz}$ and $\mathrm{Pz}\left(\mathrm{Cz}>\right.$ mean Fz/Pz: $\left.F=18.88, p<0.001, \eta_{p}^{2}=0.33\right)$. Latencies were longer for targets than nontargets (target $>$ nontarget: $F=$ $\left.8.79, p=0.005, \eta_{p}^{2}=0.18\right)$, and this delay was most apparent at $\mathrm{Cz}$ (target $>$ nontarget $\times \mathrm{Cz}>$ mean Fz/Pz: $F=13.97, p=0.001$, $\eta_{p}^{2}=0.26$ ). Figure 4 (middle) shows $\mathrm{P} 3$ latencies to targets over TTI for the young and older groups separately at Cz. Latencies were longer for the older compared to the young group (older $>$ young: $\left.F=7.07, p=0.011, \eta_{p}^{2}=0.15\right)$. There was a trend toward 


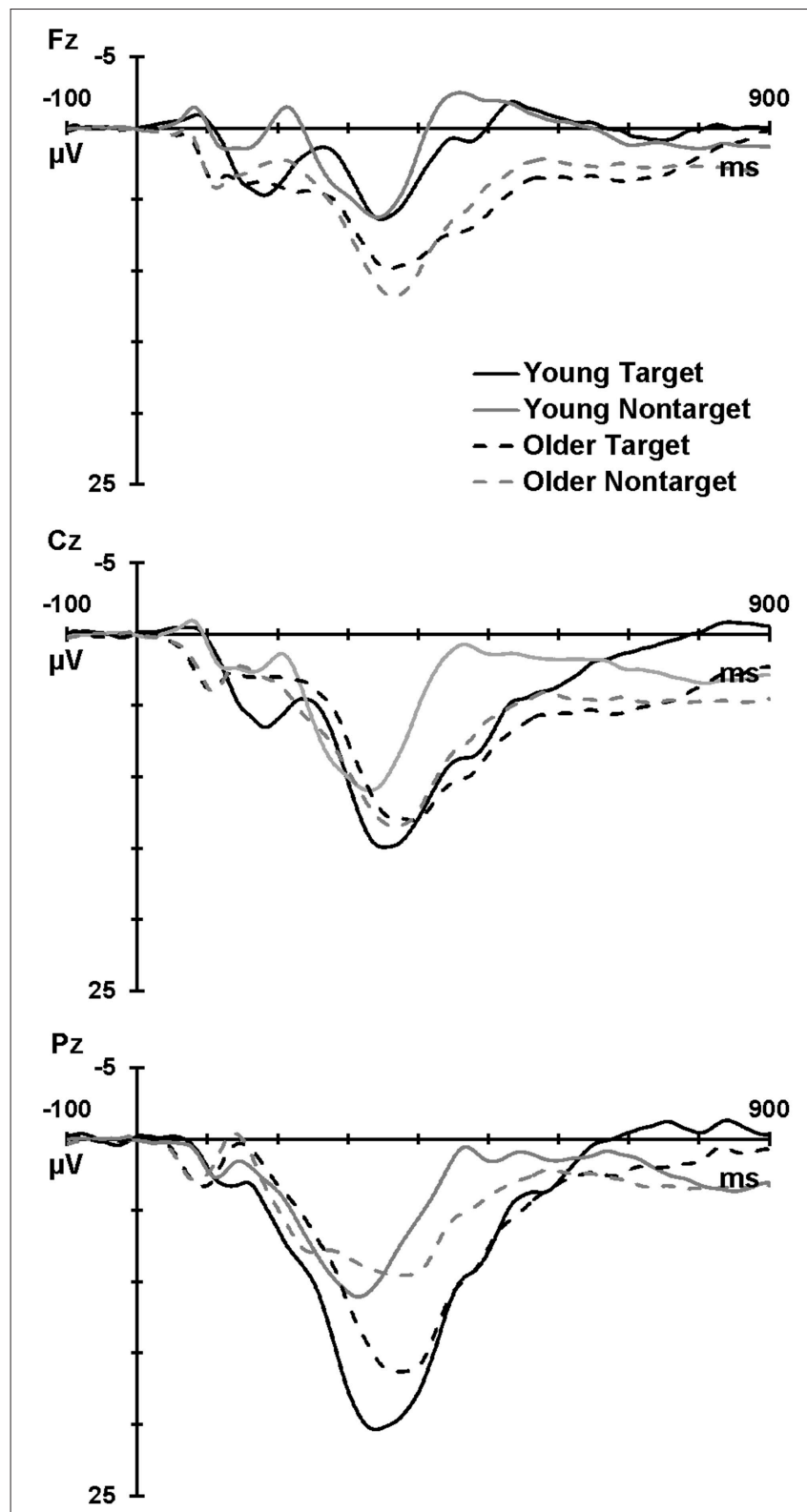

FIGURE 1 | Grand mean ERPs at Fz, Cz, and Pz for targets (black) and nontargets (gray) for young (solid line) and older (dashed line) adults.

a decrease in latency with increases in TTI at Cz (linear TTI $\times \mathrm{Cz}$ $>$ mean Fz/Pz: $\left.F=3.36, p=0.074, \eta_{p}^{2}=0.08\right)$, this did not differ between young and older adults; see Figure 4 (middle).

\section{RT}

As shown in Figure 4 (lower panel), there was a trend toward slower RTs in the older compared to the young group (young $<$ older: $\left.F=2.87, p=0.098, \eta_{p}^{2}=0.07\right)$. TTI significantly affected RT, with faster RTs to longer TTIs (linear TTI: $F=15.91, p<$ $\left.0.001, \eta_{p}^{2}=0.29\right)$. This did not differ statistically with group, but Table 1 shows a more consistent relative decrease in RT for older compared to young adults.

\section{Correlations}

Three separate bivariate correlations compared P3 amplitudes with latencies and RT, and P3 latencies with RT. There was no significant association for any of these 3 comparisons for either group, although there was a trend toward an inverse relationship between P3 amplitudes and RT for young adults, $r_{(74)}=-0.159$, $p=0.086$, one-tailed, and between P3 latencies and RT, $r_{(74)}=$ $-0.153, p=0.094$. Fisher transformations revealed no significant differences for any of these comparisons for young compared to older adults.

\section{DISCUSSION}

This work compared TTI effects on the P3 in older and young adults. Results were in line with expectations that young adults would show a greater TTI effect than older adults. These findings suggest that older adults have weaker electrophysiological memory-updating processes than young adults, as interpreted by the template-update model.

\section{TTI Effects and Memory-Updating}

P3 amplitudes were augmented by longer TTIs in both young and older adults; a finding in line with all previous TTI studies (Gonsalvez et al., 1999, 2007; Gonsalvez and Polich, 2002; Croft et al., 2003; Steiner et al., 2013a,b; Steiner et al., 2014a,b). As hypothesized, the linear increase in P3 amplitude with longer TTIs differed with age, with young adults showing a steeper increase than older adults. The relative change in P3 amplitudes ( $c f$. the first TTI level) clearly demonstrates this with amplitudes increasing to $131 \%$ of their initial value by the longest TTI for young adults, but increasing to only $113 \%$ for older adults by the second TTI level, before stabilizing at that magnitude. These findings are consistent with the notion that the P3 component captures updating processes following degradation of target templates in working memory, as the template-update model suggests (Gonsalvez et al., 2007), and that this process is compromised in older adults.

From the template-update perspective, the practice of averaging P3 amplitudes across TTIs is flawed because P3 represents an update process that can be meaningfully captured only by a function across time (as shown in Figures 2, 4). Because updating processes can be affected by several factors including encoding strengths (e.g., salient stimuli, stimuli of higher intensities, and stimuli that activate networks with rich associations) and differential slopes of template decay, P3 values at longer TTIs (especially if the value demonstrates the peak reaching asymptote) are of significance, because the value represents the completion of updating and a potential measure of encoding strength. On the other hand P3 values during intermediate TTIs could represent the effect of two or more processes (stimulus encoding strength and decay). Within this context, smaller amplitudes at longer TTIs (e.g., 9 s) would suggest inadequate encoding (weaker encoding, fewer or less effective activation of associative networks) of target stimuli in the older group. These results are consistent with literature suggesting that the ability to maintain templates 

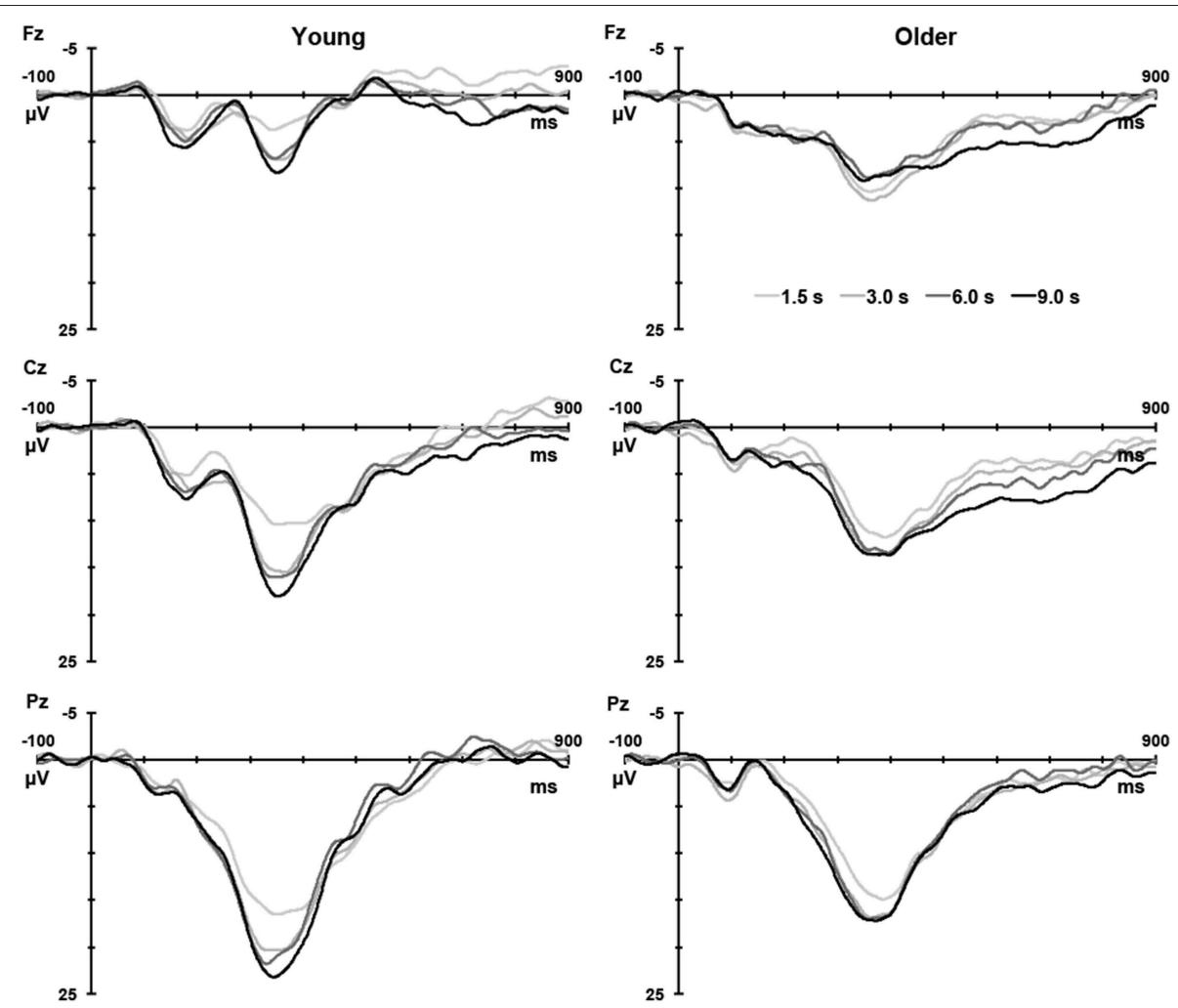

FIGURE 2 | Grand mean ERPs to targets for each analyzed TTI (1.5, 3.0, 6.0, and 9.0 s) at Fz, Cz, and Pz for young (left) and older (right) adults.

required for stimulus categorization decreases with age (Fabiani and Friedman, 1995).

Both decreased P3 amplitudes and increased P3 latencies are reliable predictors of decline of cognitive processing in aging and dementia. The current study clarifies these results by demonstrating that the indices that best differentiate the two groups are P3 amplitudes derived from longer TTIs (templateencoding differences) and P3 latencies at shorter TTIs (delayed stimulus categorization or recovery functions for template formation). In addition, RTs were faster with increases in TTI, and there was a trend toward the same pattern of results for P3 latency across both age groups; a finding consistent with previous work (Gonsalvez and Polich, 2002; Gonsalvez et al., 2007; Steiner et al., 2013b). There were no significant correlations between P3 amplitudes and latencies, between P3 amplitudes and RTs, or between P3 latencies and RTs for both groups, suggesting that P3 amplitude over TTI is an independent index of memoryupdating processes.

Older adults showed an overall reduction in processing power and longer processing times compared to young adults. This was evidenced by reduced P3 amplitudes, longer P3 latencies, and a trend toward slower RTs in older vs. young adults. These are well-established findings (Polich, 1997; West et al., 2010; Barry et al., 2016) that are also accompanied by an anterior shift in P3 topography (evident in Figure $\mathbf{1}$ at $\mathrm{Fz}$ and in Figure 3) due to a reduction in processing in the parietal cortex and an increased reliance on hippocampus and prefrontal

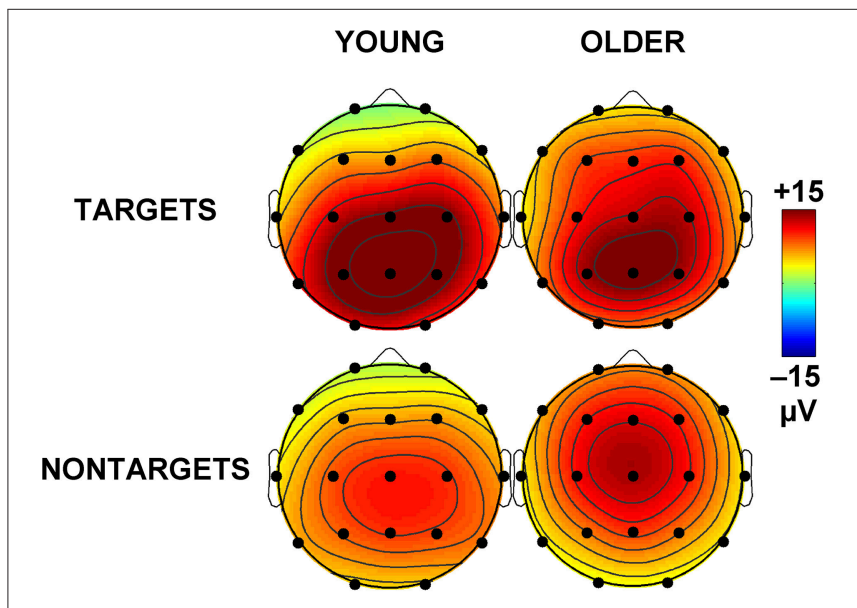

FIGURE 3 | Mean topographic headmaps for each stimulus category (top: targets; bottom: nontargets) and each group (left: young adults at $350 \mathrm{~ms}$; right: older adults at $365 \mathrm{~ms}$ ).

structures including dorsolateral prefrontal cortex (O'Connell et al., 2012). This compensatory processing also takes more time, resulting in slower RTs (Falkenstein et al., 2006). Our pattern of results is consistent with the Scaffolding Theory of Cognitive Ageing, where neuroplasticity alleviates the pathophysiological cognitive decline associated with aging (Goh and Park, 2009). Here, the brain responds to age-related changes in anatomy and 


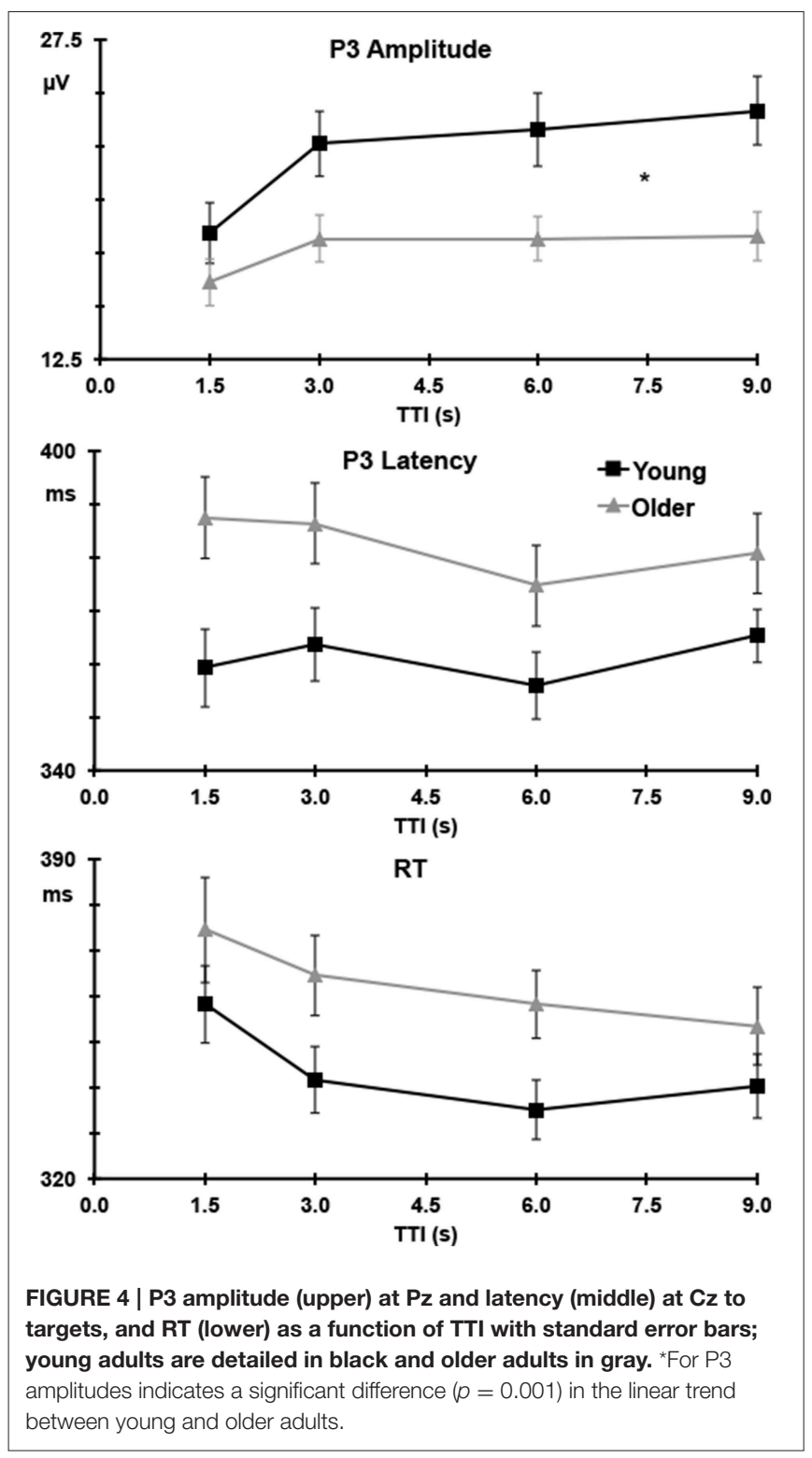

physiology (e.g., reductions in cortical thickness, dopaminergic activity, white matter integrity) by recruiting additional regions of activation and increasing its activity in order to meet the demands of information processing. This is evidenced in our study as both groups responded with similar task accuracy (2.12 vs. $2.02 \%$ errors), but the older group showed a more diffuse distribution of P3 amplitudes (see Figure 3; positivity indicated in red is distributed more broadly across the scalp).

\section{Limitations}

Because the primary focus was to study the effects of TTI as a function of age, TTI manipulations using a single stimulus were considered. Such a paradigm was trialed initially but abandoned because several subjects, especially older adults, tended to become drowsy when targets were separated by long intervals of silence (e.g., 9 s). The task structure utilized in the current study could be optimized in future work. Paradigms using longer TTIs (e.g., $15 \mathrm{~s}$; Steiner et al., 2013a) are more sensitive for capturing memory-updating differences and would be more suitable as potential predictors of aging and dementia. In addition, this two-stimulus task with a fixed ISI meant that TTI was confounded with stimulus-sequence. Future work could disentangle these effects by utilizing a three-stimulus task, or randomly varying the ISI around a $1.5 \mathrm{~s}$ mean. Further research examining the relationship between cognitive measures and the various subcomponents of the P3 is also warranted. It should also be noted that we did not obtain the level of education of participants, however all participants were well functioning, understood the task instructions (as indicated by their high performance), and could read and write in order to provide informed consent.

\section{CONCLUSION}

This study examined TTI effects on the P3 in young and older adults. We found that increases in TTI enhanced P3 amplitude in a linear fashion, and this effect was weaker for older adults. There was also an overall decrease in P3 amplitudes, a slowing of latencies and RTs, and an anterior shift in topography in older adults. Findings indicate that memory-updating processes are compromised in older adults, with a poorer ability to encode, maintain, and update stimulus templates in working memory. Further work is needed to ascertain whether people living with dementia or Mild Cognitive Impairment (MCI) show a different pattern of results.

\section{AUTHOR CONTRIBUTIONS}

GS analyzed the data and drafted the manuscript. CG designed the study, liaised with the aged care facility, supervised the project, and provided critical feedback on the drafted manuscript. FD collected the data. RB supervised the project and provided critical feedback on the drafted manuscript.

\section{FUNDING}

Thank you to the Illawarra Health and Medical Research Institute (IHMRI) for funding this research.

\section{ACKNOWLEDGMENTS}

We thank The Illawarra Retirement Trust (IRT) and the management of IRT Links Seaside for their kind assistance in providing facilities and encouraging resident participation in this project. In addition, we would like to acknowledge the Illawarra Health and Medical Research Institute (IHMRI) for funding this research. 


\section{REFERENCES}

Barry, R. J., De Blasio, F. M., and Cave, A. E. (2016) Sequential processing in young and older adults in the equiprobable auditory Go/NoGo task. Clin. Neurophysiol. 127, 2273-2285. doi: 10.1016/j.clinph.2016.02.010

Bishop, N. A., Lu, T., and Yankner, B. A. (2010). Neural mechanisms of ageing and cognitive decline. Nature 464, 529-535. doi: 10.1038/nature08983

Conforti, D., Rowland, J, Basic, D., Vrantsidis, F., Hill, K., LoGiudice, D., et al. (2006). A Study to Evaluate the Rowland Universal Dementia Assessment Scale (RUDAS) in Two Populations Outside of the Sydney South West Area Health Service.

Croft, R. J., and Barry, R. J. (2000). Removal of ocular artifact from the EEG: a review. Neurophysiol. Clin. 30, 5-19. doi: 10.1016/S0987-7053(00)00055-1

Croft, R. J., Gonsalvez, C. J., Gabriel, C., and Barry, R. J. (2003). Target-totarget interval versus probability effects on P300 in one- and two-tone tasks. Psychophysiology 40, 322-328. doi: 10.1111/1469-8986.00036

Fabiani, M., and Friedman, D. (1995). Changes in brain activity patterns in aging: the novelty oddball. Psychophysiology 32, 579-594.

Falkenstein, M., Yordanova, J., and Kolev, V. (2006). Effects of aging on slowing of motor-response generation. Int. J. Psychophysiol. 59, 22-29. doi: 10.1016/j.ijpsycho.2005.08.004

Goh, J. O., and Park, D. C. (2009). Neuroplasticity and cognitive aging: the scaffolding theory of aging and cognition. Restor. Neurol. Neurosci. 27, 391-403. doi: 10.3233/RNN-2009-0493

Gonsalvez, C. J., and Polich, J. (2002). P300 amplitude is determined by target-totarget interval. Psychophysiology 39, 388-396. doi: 10.1017/S0048577201393137

Gonsalvez, C. J., Barry, R. J., Rushby, J. A., and Polich, J. (2007). Target-totarget interval, intensity, and P300 from an auditory single-stimulus task. Psychophysiology 44, 245-250. doi: 10.1111/j.1469-8986.2007.00495.x

Gonsalvez, C. J., Gordon, E., Grayson, S., Barry, R. J., Lazzaro, I., and Bahramali, H. (1999). Is the target-to-target interval a critical determinant of P3 amplitude? Psychophysiology 36, 643-654.

Hedden, T., and, Gabrieli, J. D. E. (2004). Insights into the ageing mind: a view from cognitive neuroscience. Nat. Rev. Neurosci. 5, 87-96. doi: $10.1038 / \mathrm{nrn} 1323$

Hester, R. L., Kinsella, G. J., and Ong, B. (2004).Effect of age on forward and backward span tasks. J. Int. Neuropsychol. Soc. 10, 475-481. doi: $10.1017 / \mathrm{s} 1355617704104037$

Howell, D. C. (1997). Statistical Methods for Psychology. Boston, MA: Duxbury Press.

Kadota, T., Horinouchi, T., and Kuroda, C. (2001). Development and aging of the cerebrum: assessment with proton MR spectroscopy. AJNR. Am. J. Neuroradiol. 22, 128-135. Available online at: http://www.ajnr.org/content/22/1/128.full

O'Connell, R. G., Balsters, J. H., Kilcullen, S. M., Campbell, W., Bokde, A. W., Lai, R., et al. (2012). A simultaneous ERP/fMRI investigation of the P300 aging effect. Neurobiol. Aging 33, 2448-2461. doi: 10.1016/j.neurobiolaging.2011.12.021

Pieperhoff, P., Homke, L., Schneider, F., Habel, U., Shah, N. J., Zilles, K., et al. (2008). Deformation field morphometry reveals age-related structural differences between the brains of adults up to 51 years. J. Neurosci. 28, 828-842. doi: 10.1523/JNEUROSCI.3732-07.2008

Polich, J. (1997). On the relationship between EEG and P300: individual differences, aging, and ultradian rhythms. Int. J. Psychophysiol. 26, 299-317. doi: 10.1016/s0167-8760(97)00772-1

Salat, D. H., Lee, S. Y., Van der Kouwe, A. J., Greve, D. N., Fischl, B., and Rosas, H. D., (2009). Age-associated alterations in cortical gray and white matter signal intensity and gray to white matter contrast. NeuroImage 48, 21-28. doi: 10.1016/j.neuroimage.2009.06.074

Squires, K. C., Wickens, C., Squires, N. K., and Donchin, E. (1976). The effect of stimulus sequence on the waveform of the cortical event-related potential. Science 193, 1142-1146. doi: 10.1126/science.959831

Squires, K., Petuchowski, S., Wickens, C., and Donchin, E. (1977). The effects of stimulus sequence on event related potentials: a comparison of visual and auditory sequences. Percept. Psychophys. 22, 31-40. doi: 10.3758/BF03206077

Steiner, G. Z., Barry, R. J., and Gonsalvez, C. J. (2013a). Can working memory predict target-to-target interval effects in the P300? Int. J. Psychophysiol. 89, 399-408. doi: 10.1016/j.ijpsycho.2013.07.011

Steiner, G. Z., Barry, R. J., and Gonsalvez, C. J. (2014a). Stimulus-to-matchingstimulus interval influences N1, P2, and P3b in an equiprobable Go/NoGo task. Int. J. Psychophysiol. 94, 59-68. doi: 10.1016/j.ijpsycho.2014.07.003

Steiner, G. Z., Barry, R. J., and Gonsalvez, C. J. (2014b). Nontarget-tonontarget interval determines the nontarget P300 in an auditory equiprobable Go/NoGo task. Int. J. Psychophysiol. 92, 113-121. doi: 10.1016/j.jpsycho.2014. 03.010

Steiner, G. Z., Brennan, M. L., Gonsalvez, C. J., and Barry, R. J. (2013b). Comparing P300 modulations: Target-to-target interval versus infrequent nontarget-tonontarget interval in a three-stimulus task. Psychophysiology 50, 187-194. doi: 10.1111/j.1469-8986.2012.01491.x

Tabachnick, B. G., and Fidell, L. S. (1989). Using Multivariate Statistics. New York, NY: Harper Collins.

Verleger, R. (1991). The instruction to refrain from blinking affects auditory P3 and N1 amplitudes. Electroencephalogr. Clin. Neurophysiol. 78, 240-251. doi: 10.1016/0013-4694(91)90039-7

West, R., Schwarb, H., and Johnson, B. N. (2010). The influence of age and individual differences in executive function on stimulus processing in the oddball task. Cortex 46, 550-563. doi: 10.1016/j.cortex.2009. 08.001

Conflict of Interest Statement: The authors declare that the research was conducted in the absence of any commercial or financial relationships that could be construed as a potential conflict of interest.

Copyright (c) 2016 Steiner, Gonsalvez, De Blasio and Barry. This is an open-access article distributed under the terms of the Creative Commons Attribution License (CC $B Y)$. The use, distribution or reproduction in other forums is permitted, provided the original author(s) or licensor are credited and that the original publication in this journal is cited, in accordance with accepted academic practice. No use, distribution or reproduction is permitted which does not comply with these terms. 